Article

\title{
Essential Oil Composition of Artemisia herba-alba from Southern Tunisia
}

\section{Haouari Mohsen * and Ferchichi Ali \\ Laboratoire d'Aridoculture et Culture Oasienne, Institut des Régions Arides 4119 Medenine, Tunisia; E-mail: Ferchichi.ali@ira.rnrt.tn (F.A.)}

* Author to whom correspondence should be addressed; E-mail: Haouari.M@gmail.com.

Received: 19 January 2009; in revised form: 27 February 2009 / Accepted: 9 March 2009 /

Published: 20 April 2009

\begin{abstract}
The composition of the essential oil hydrodistilled from the aerial parts of 18 individual Artemisia herba-alba Asso. plants collected in southern Tunisia was determined by GC and GCMS analysis. The oil yield varied between $0.68 \% \mathrm{v} / \mathrm{w}$ and $1.93 \% \mathrm{v} / \mathrm{w}$. One hundred components were identified, 21 of of which are reported for the first time in Artemisia herba-alba oil. The oil contained 10 components with percentages higher than $10 \%$. The main components were cineole, thujones, chrysanthenone, camphor, borneol, chrysanthenyl acetate, sabinyl acetate, davana ethers and davanone. Twelve samples had monoterpenes as major components, three had sesquiterpenes as major components and the last three samples had approximately the same percentage of monoterpenes and sesquiterpenes. The chemical compositions revealed that ten samples had compositions similar to those of other Artemisia herba-alba essential oils analyzed in other countries. The remaining eight samples had an original chemical composition.
\end{abstract}

Keywords: Herba-alba; Asteraceae; Essential oil composition; Chemotypes.

\section{Introduction}

Artemisia herba-alba Asso. known also as "desert wormwood" is a prominent plant of the IranoTuranien steppes of Spain, North Africa and the Middle East [1-4]. It is one of five spontaneous Artemisia species recorded in Tunisia [5]. This plant is used as aromatisant for tea and in folk 
medicine for treatment of colds, coughing, intestinal disturbances and as antidiabetic agent [6, 7]. A survey carried out among the South Tunisian population showed that Artemisia herba-alba is the most recognized aromatic and medicinal plant [8]. In Tunisia, this greenish-silver perennial shrub is present in areas with between $400 \mathrm{~mm}$ and $90 \mathrm{~mm}$ of annual precipitations. It is characterized by its drought tolerance, essentially due to its leaves' polymorphism and root architecture [9-11].

Investigations on the medicinal properties of $A$. herba-alba extracts reported anti-diabetic, leishmanicidal, antibacterial, and antifungal properties [12-15]. Over last decades, studies on $A$. herba-alba were focused on its essential oils. Their composition in different parts of the world revealed a high level of polymorphism and led to the definition of several chemotypes.

Studies from Spain $[16,17]$ showed that monoterpene hydrocarbons and oxygenated monoterpenes are the most abundant skeletons in A. herba-alba oil, but large amounts of sesquiterpenes were found for some populations. Camphor, 1,8-cineole, $p$-cymene and davanone were the major compounds found. Two oil types were found for plants grown in Israel and Sinai [18] those of cineol-thujanebornane type and the pinane type with monoterpene skeletons. Chemotaxonomic affinity between Spanish and Israeli populations of the same plant was not reflected in their oil composition.

In Jordan regular monoterpenes were predominant and the principal components were $\alpha$ - and $\beta$ thujones, classifyng the plant as being a thujone chemotype [19]. In Morocco, the market leader in $A$. herba-alba essential oil exports, 16 chemotypes were found [20], with 12 having monoterpenes as major components and for four, sesquiterpene skeletons represent the major fraction of the oil. Investigations reported no correlation between chemotypes and geographic distribution. [21]

For Algerian oil monoterpenes were the major components, essentially camphor, $\alpha$ - and $\beta$-thujones, 1,8-cineole and chrysanthenyl derivatives [22,23]. In Tunisian oil oxygenated monoterpenes were found to be the major components of $A$. herba-alba oil extracted from aerial parts of plants originated from arid regions $[24,25]$.

Studies on A. herba-alba didn't cover the entire domain where this plant is found. The aim of this study was to elucidate the chemical polymorphism of A. herba-alba from the sub-arid to Saharan domains in Tunisia.

\section{Results and Discussion}

In order to study the chemical composition and variability of the A. herba-alba essential oils, 18 individual plants were collected from different populations located in Southern Tunisia. These plants were selected from environmentally diverse areas and subcultured under the same ecological conditions.

Hydrodistillation of the aerial parts of these A. herba-alba samples yielded yellowish liquid oils. The oil yield varied between $0.68 \% \mathrm{v} / \mathrm{w}$ and $1.93 \% \mathrm{v} / \mathrm{w}$, based on dried weight of samples. Same variation in the essential oil yield was also found in Spain [17].

The chemical composition of the oil was investigated using both GC and GC-MS techniques. Table 1 lists the components identified in the essential oil of $A$. herba-alba, their RI, and their concentration in the different samples. One hundred components, accounting for $93.5 \%$ to $100 \%$ of the oil, were identified, 75 of them being reported for the first time in Tunisian oil, to our knowledge, and 21 of which have not been previously reported in A. herba-alba oils (see footnotes in Table 1). Among the 
18 essential oil samples, monoterpenes constituted the major fraction of the oil in 12 samples, amounting to more than $57 \%$ of the total oil (Table 1). In three samples (E2, E10, and E14), sesquiterpenes were more abundant than monoterpenes, with concentrations as high as $54 \%$ of the total oil. For the last three samples (E3, E6, and E13), monoterpenes and sesquiterpenes have sensibly the same contribution to the total oil composition (Table 1). For monoterpene-rich samples, oxygenated monoterpenes are the most abundant and for sesquiterpene-rich samples, oxygenated sesquiterpenes represent the major fraction of the oil.

Among the hundred identified components, ten of them (cineole, thujones, chrysanthenone, camphor, borneol, chrysanthenyl acetate, sabinyl acetate, davana ethers and davanone) could be considered as major components, with concentrations superior to $10 \%$ of the total oil.

Cineole was reported as the major component in the essential oil from Spain [16, 17], Israel [18], Egypt [26], and Morocco [20]. Its concentration reaches high values (superior to 40\%) in Spain and Israel $[17,18]$. In Tunisian A. herba-alba oil, previous studies reported none or very low values for 1,8-cineole concentration [24,25], like essential oils from Algeria and Jordan [19, 23]. In this study 1,8-cineole concentration varied between $0.6 \%$ in sample E2 and $26 \%$ in sample E18. This kind of variation was observed in Moroccan oil where a cineole-camphor chemotype was defined [20].

Thujones were absent in two samples (E2 and E3) but represented 64\% of the E15 sample. These components give the specific odor and taste to A. herba-alba plants. The absence of thujones was reported for all the oils from Spain $[16,17]$. Reported concentration in the other oils varies, but didn't reach the concentration observed for the E15 sample in this study.

Chrysanthenone reached the highest percentage (17\%) in sample E2. This component, with a pinane skeleton, wasn't found in Tunisian A. herba-alba essential oil at such high concentrations $[24,25]$. In Spain and Algeria, chrysanthenone was found at amounts comparable to those found in this study [17,23]. In Morocco, a chrysanthenyl chemotype was defined, with chrysanthenone concentrations as high as $77 \%$ of the total oil [20].

Camphor was found at high concentrations in five samples (E1, E11, E12, E17, and E18) and constituted the major component in two of them (E1 and E12). This chemical is one of the most encountered components in A. herba-alba oil. In Morocco, five chemotypes were defined as camphor type oils [20].

Borneol was found as a major component in sample E18. High levels of borneol were found in oils from the Sinai [18], but the chemotype defined there (cineole-thujone-borneol) is characterized by its high level of thujone, whereas sample E18 has a low level of thujone but a high camphor concentration.

Chrysanthenyl acetate was found at its highest concentrations in sample E9 with sabinyl acetate and thujones as main components. Such association was observed in A. herba-alba from Tunisia [24].

Sabinyl acetate was present as a major component in five samples, with a concentration range from $10 \%$ to $22 \%$ of the total oil. High levels of sabinyl acetate were only found in oils originated from the south of Tunisia [8,24,27].

The main sesquiterpenes found in the studied oils are davana ethers and davanone. Several davana ether isomers were identified. In Tunisia (this study) three isomers were found, while in Spain two isomers were identified [17], and in Morocco four isomers were described in A. herba-alba oil [20]. 
Davanone, and its derivatives, were also identified in Israel [18], but the highest levels were observed in Morocco, leading to the definition of four davanone chemotypes [20].

Of the 18 samples studied ten have compositions similar to published essential oils of $A$. herbaalba. Sample E4 has thujones and sabinyl acetate as main components as the oil extracted from other locations in Tunisia [24]. Samples E6, E7, E8, E14, E15, and E16, with thujones as major components, have their equivalent in Moroccan and Jordanian oils [19, 20]. A thujones-camphor oil (sample E12) was also observed in Morocco [20] and cineole-camphor-borneol oil (sample E18) was observed in Israel [18]. The most frequent chemotype is composed of cineol and camphor and was observed in sample E17 and in Morocco, Spain and Israel [16,18,20].

The eight remaining samples (E1, E2, E3, E5, E9, E10, E11, and E13) have an original composition. The main components association observed in these oils was not observed in A. herbaalba essential oil composition published elsewhere.

Table 1. Chemical composition of essential oils of Artemisia herba-alba.

\begin{tabular}{|c|c|c|c|c|c|c|c|c|c|c|c|c|c|c|c|c|c|c|c|}
\hline Name & RI & E1 & $\mathrm{E} 2$ & E3 & E4 & E5 & E6 & E7 & E8 & E9 & E10 & E11 & E12 & E13 & E14 & E15 & E16 & E17 & E18 \\
\hline 2-Hexanal ${ }^{\mathrm{a}}$ & 841 & 0.22 & - & 0.28 & 0.35 & 0.27 & - & 0.2 & 0.23 & - & 0.28 & 0.53 & 0.22 & - & - & 0.74 & - & 0.36 & - \\
\hline$n$-Hexanol ${ }^{\mathrm{a}}$ & 860 & - & - & 0.26 & - & - & - & - & - & - & 0.23 & - & - & - & - & - & - & - & - \\
\hline Geraniolene $^{\mathrm{a}}$ & 880 & - & - & - & - & - & - & 0.13 & - & - & 0.13 & - & - & - & - & - & - & - & - \\
\hline Santolina triene & 909 & - & - & 0.23 & - & 0.21 & - & - & - & - & 0.14 & - & - & 0.14 & - & - & - & 0.52 & - \\
\hline 2.5-Diethenyl-2-methyltetrahydrofuran & 916 & - & 0.39 & 0.47 & 0.28 & 0.53 & 0.32 & - & 0.33 & 0.21 & 0.35 & - & - & 0.36 & - & - & 0.15 & - & - \\
\hline Tricyclene & 924 & - & - & - & - & - & - & - & - & - & - & 0.28 & 0.29 & - & - & - & - & 0.31 & 0.34 \\
\hline$\alpha$-Pinene & 933 & 0.2 & - & 0.27 & - & 0.16 & 0.17 & 0.35 & 0.61 & - & 0.22 & - & 0.62 & - & 0.13 & - & 0.34 & - & - \\
\hline Unknown 1 & 940 & - & 0.43 & 0.54 & 0.37 & 0.65 & 0.42 & - & 0.33 & - & 0.38 & - & - & 0.46 & - & - & 0.22 & - & - \\
\hline Camphene & 949 & 1.64 & 0.28 & 0.31 & - & 0.25 & 0.95 & 1.44 & 0.51 & 0.26 & 0.35 & 2.42 & 2.45 & - & - & - & 0.34 & 2.04 & 2.08 \\
\hline 5.5-Dimethyl-2 $(5 \mathrm{H})$-furanone & 952 & - & 0.37 & 0.59 & - & - & - & - & - & - & - & - & - & - & 0.44 & - & - & - & - \\
\hline Sabinene & 971 & - & 0.17 & 0.12 & 0.67 & - & 0.2 & - & 0.4 & - & 0.17 & - & 1.5 & 0.48 & - & 1.14 & 0.51 & - & - \\
\hline$\beta$-Pinene & 978 & 1.15 & - & - & - & 0.91 & - & - & - & - & - & - & 0.42 & - & - & - & - & - & - \\
\hline 1-Octen-3-ol & 983 & - & - & - & - & - & - & - & - & - & - & - & - & - & - & - & - & - & 1.03 \\
\hline$\beta$-Myrcene & 994 & - & - & 0.42 & - & 0.4 & - & 0.56 & - & - & 0.46 & - & 0.4 & - & - & - & 0.5 & - & - \\
\hline$\alpha$-Phellandrene & 998 & - & - & - & - & - & - & - & - & - & - & - & - & - & - & - & 0.21 & - & - \\
\hline Yomogi alcohol & 999 & - & - & - & - & 0.18 & - & - & - & - & - & - & - & - & - & - & - & 3.72 & 9.46 \\
\hline 2-Carene ${ }^{\mathrm{a}}$ & 1001 & 1.16 & - & - & - & 0.2 & - & - & - & - & - & - & - & - & - & - & - & - & - \\
\hline$\alpha$-Terpinene & 1012 & - & - & - & - & - & - & - & 0.23 & - & - & - & 0.34 & - & - & 0.53 & 0.29 & - & - \\
\hline$p$-Mentha-1(7).8-diene & 1018 & - & 0.25 & 0.31 & - & 0.36 & - & - & - & - & 0.25 & - & - & 0.25 & - & - & - & - & - \\
\hline$p$-Cymene & 1025 & - & - & 0.35 & 0.79 & 0.7 & 0.67 & 2.3 & 0.85 & 0.82 & - & 2.18 & - & 0.65 & 0.3 & 2.77 & 1.39 & - & - \\
\hline Eucalyptol & 1032 & 11.18 & 0.61 & 2.68 & 2.58 & 2.14 & 4.88 & 5.22 & 2.46 & 4.24 & 1.52 & 10.19 & 6.89 & 1.57 & 1.07 & 7.33 & 4.13 & 18.35 & 26.99 \\
\hline Santolina alcohol & 1038 & - & - & - & - & - & - & - & - & - & - & - & - & - & - & - & - & 3.71 & 3.37 \\
\hline cis-Arbusculone & 1052 & - & 2.98 & 4.24 & - & - & - & 1.96 & 2.81 & 1.09 & 4.58 & - & - & 1.75 & 0.91 & - & 0.9 & - & - \\
\hline$\gamma$-Terpinene & 1062 & 1.92 & - & 0.28 & 0.48 & 0.45 & 0.22 & - & 0.42 & - & - & - & 0.67 & 0.31 & - & 1.2 & 0.4 & 0.47 & 0.53 \\
\hline Artemisia ketone & 1064 & - & - & - & - & - & - & - & - & - & - & - & - & - & - & - & - & 2.65 & - \\
\hline cis-Sabinene hydrate & 1070 & 2.08 & - & - & - & - & - & - & - & - & - & - & - & - & - & - & - & - & - \\
\hline trans-Arbusculone & 1072 & - & 2.27 & 3.53 & - & - & - & 1.48 & 2.35 & 0.92 & 3.6 & - & - & 1.65 & 0.72 & - & 0.75 & - & - \\
\hline Artemisia alcohol & 1084 & - & - & - & - & - & - & - & - & - & - & - & - & - & - & - & - & 2.05 & 2.97 \\
\hline Terpinolene & 1088 & 0.75 & - & - & - & - & - & - & - & - & 0.28 & - & - & - & - & - & - & - & - \\
\hline Isochrysanthenone & 1098 & - & 2.95 & 2.09 & - & - & - & - & - & - & 3.96 & - & - & - & - & - & - & - & - \\
\hline
\end{tabular}


Table 1. Cont.

\begin{tabular}{|c|c|c|c|c|c|c|c|c|c|c|c|c|c|c|c|c|c|c|c|}
\hline$\alpha$-Thujone & 1110 & 11.54 & - & - & 34.32 & 8.2 & 12.85 & 20.04 & 5.99 & 16.38 & 0.8 & 23.16 & 16.51 & 11.63 & 13.39 & 42.23 & 11.36 & 4.58 & 2.11 \\
\hline$\beta$-Thujone & 1114 & 6.03 & - & - & 4.41 & 5.48 & 2.18 & 10.88 & 6.96 & 9.57 & - & 24.05 & 11.18 & 5.88 & 5.06 & 22.44 & 3.4 & 5.74 & 1.28 \\
\hline cis-p-Menth-2-en-1-ol & 1118 & 2.19 & - & - & 0.77 & - & - & - & 1.04 & - & - & - & - & 0.6 & - & - & - & 2.15 & - \\
\hline Chrysanthenone & 1123 & - & 17.37 & 7.89 & - & 10.08 & 4.83 & 8.97 & 1.07 & 9.02 & 11.65 & 1.15 & 8.64 & - & 6.19 & 0.91 & 8.34 & 2.69 & 3.8 \\
\hline trans-Pinocarveol & 1136 & - & 0.63 & 0.9 & 0.56 & 0.34 & - & - & 5.39 & 2.68 & 0.62 & - & - & 0.7 & 2.76 & 1.79 & 2.58 & - & - \\
\hline$\delta$-Verbenol ${ }^{\mathrm{a}}$ & 1141 & - & 0.68 & 4.58 & - & - & - & - & - & 2.12 & 0.34 & - & - & - & - & - & 3.2 & - & - \\
\hline cis-Sabinol & 1143 & - & - & - & 5.94 & 1.64 & - & - & 2.4 & - & - & - & - & 2.49 & - & - & - & - & - \\
\hline Camphor & 1144 & 13.25 & - & - & 0.56 & - & 5.96 & 7.05 & - & - & 0.63 & 16.73 & 17.81 & - & 0.61 & 2.54 & - & 17.13 & 12.85 \\
\hline Ssabina ketone & 1156 & - & - & - & - & - & - & - & - & 0.37 & - & 0.73 & - & 0.24 & - & 0.53 & - & - & - \\
\hline Pinocarvone & 1160 & 1.07 & - & - & - & - & 0.32 & 0.4 & 0.68 & 0.41 & - & 0.77 & 1.2 & - & 0.39 & 0.83 & 0.73 & 0.91 & 0.95 \\
\hline Borneol & 1168 & 1.91 & - & 0.72 & - & - & 2.14 & 1.61 & 1.13 & 0.89 & - & 4.11 & 5.24 & - & 0.55 & 0.69 & 0.91 & 4.94 & 10.75 \\
\hline$\alpha$-Phellandren-8-ol ${ }^{\mathrm{a}}$ & 1170 & - & - & 1.17 & - & - & - & - & - & - & - & - & - & - & - & - & - & - & - \\
\hline Lavandulol & 1170 & - & - & - & - & - & - & - & - & - & - & - & - & - & - & - & - & 1.44 & - \\
\hline 4-Terpineol & 1177 & 8.71 & 0.98 & 0.89 & 3.38 & 1.93 & 1.7 & 1.81 & 2.12 & 0.57 & 1 & 1.5 & 2.65 & 1.24 & 1.75 & 3.4 & 1.7 & 2.5 & 2.08 \\
\hline$\alpha$-Thujenal $^{\mathrm{a}}$ & 1182 & 0.72 & - & - & - & - & - & - & 0.33 & - & - & - & - & 0.4 & - & - & 0.43 & - & - \\
\hline p-Cymen-8-ol & 1184 & - & - & - & - & - & - & - & - & - & - & - & - & - & - & - & - & - & 1.14 \\
\hline$\alpha$-Terpineol & 1189 & 1 & - & - & - & - & - & - & - & - & - & - & 0.62 & - & 0.31 & - & - & - & 1.02 \\
\hline cis-Piperitol & 1194 & - & - & - & - & - & - & - & - & 0.98 & - & - & - & - & - & - & 1.37 & - & - \\
\hline Myrtenal & 1197 & - & - & - & - & - & - & - & - & - & - & 0.73 & - & - & - & - & - & 1.26 & 1.21 \\
\hline Myrtenol & 1198 & 1.21 & - & - & - & - & 0.45 & - & 1.08 & - & - & 0.79 & 1.11 & - & 0.67 & 1.27 & - & 0.92 & 1.21 \\
\hline Verbenone & 1203 & - & - & 0.37 & - & - & - & - & - & - & - & - & - & - & - & - & - & - & - \\
\hline trans-Piperitol & 1211 & 1.15 & - & - & - & 0.71 & 0.46 & 0.69 & 0.63 & 1.19 & - & - & 1.28 & 0.46 & 0.58 & 0.62 & 1.67 & - & - \\
\hline Octyl acetate ${ }^{\mathrm{a}}$ & 1216 & - & - & - & - & - & - & - & - & - & - & - & - & - & - & - & - & - & 1.11 \\
\hline Nordavanone & 1227 & - & 0.59 & 0.35 & - & 0.54 & 0.48 & - & 0.46 & - & - & - & - & 0.78 & - & - & - & - & - \\
\hline Cuminaldehyde & 1235 & 1.63 & - & - & - & - & - & - & 0.62 & - & - & - & - & 0.73 & - & - & 0.72 & - & - \\
\hline Butanoic acid, 2-methyl- & & & & & & & & & & & & & & & & & & & \\
\hline hexyl ester ${ }^{a}$ & 1237 & - & - & - & - & - & - & - & - & - & 0.24 & - & - & - & - & - & - & - & - \\
\hline Carvone & 1239 & - & - & - & - & - & - & - & - & - & - & - & - & - & 0.31 & - & - & 0.65 & - \\
\hline Piperitone & 1253 & - & - & - & - & - & - & - & 0.66 & 4.56 & - & - & - & 0.75 & - & 0.81 & 3.43 & 1.83 & - \\
\hline Piperitone oxide ${ }^{a}$ & 1259 & - & - & - & - & - & - & - & 0.6 & - & - & - & 0.65 & 0.6 & 0.33 & - & 1.22 & - & - \\
\hline cis-Chrysanthenyl acetate & 1262 & 1.08 & 0.52 & 9.47 & - & 9.11 & 3.61 & 2.58 & 5.54 & 13 & - & 1.12 & 5.82 & 0.37 & 1.01 & 0.62 & 7.37 & 0.88 & 2.28 \\
\hline Isopiperitenone $^{\mathrm{a}}$ & 1271 & - & - & 0.24 & - & - & - & - & - & - & 0.39 & - & - & - & - & - & - & - & - \\
\hline Bornyl acetate & 1285 & 0.71 & - & - & - & - & - & 0.81 & 0.43 & - & - & 0.76 & 1.27 & - & 0.38 & - & - & 1.01 & 2.04 \\
\hline 4-Terpineol acetate & 1289 & - & - & - & - & - & - & 0.53 & - & - & - & - & - & - & - & - & - & - & - \\
\hline Sabinyl acetate & 1297 & 12.12 & - & - & 22.46 & 10.98 & 7.36 & 3.13 & 6.52 & 11.09 & 0.53 & 1.12 & - & 11.13 & - & - & 8.23 & 4.16 & 1.02 \\
\hline Myrtenyl acetate & 1302 & 0.73 & - & 0.27 & - & - & - & - & - & - & - & - & - & - & - & - & - & - & - \\
\hline Thymol & 1289 & - & - & - & - & - & - & 0.52 & 0.39 & - & 0.34 & - & - & - & - & - & 0.37 & - & - \\
\hline Carvacrol & 1305 & 0.59 & - & - & - & - & - & - & - & - & - & - & - & - & - & - & 0.57 & - & - \\
\hline Unknown 2 & 1310 & - & - & 0.34 & - & 0.4 & - & 0.83 & - & - & 0.6 & - & - & - & - & - & 0.49 & - & - \\
\hline$\alpha$-Copaene & 1376 & - & - & - & - & - & 0.44 & 0.68 & - & - & - & - & - & - & 0.31 & - & - & - & - \\
\hline Methyl cinnamate $^{\mathrm{a}}$ & 1379 & 0.77 & - & - & - & - & - & - & 0.54 & - & - & - & - & - & - & - & - & - & - \\
\hline Jasmone & 1394 & 0.79 & 2.25 & 0.69 & 0.83 & 1.11 & 0.88 & 1.75 & 0.87 & - & 0.95 & - & 0.95 & 0.9 & 1.64 & 0.75 & - & - & - \\
\hline cis-Davanafuran & 1414 & - & - & - & - & - & - & - & 0.43 & - & - & - & - & 0.49 & - & - & - & - & - \\
\hline$\alpha$-Humulene & 1454 & - & - & - & - & - & - & 1.03 & - & - & 1.14 & - & - & - & - & - & - & - & - \\
\hline
\end{tabular}


Table 1. Cont.

\begin{tabular}{|c|c|c|c|c|c|c|c|c|c|c|c|c|c|c|c|c|c|c|c|}
\hline Ethyl cinnamate & 1464 & 2.47 & - & - & - & - & - & 1.82 & - & - & - & 3 & - & - & - & 1.03 & - & 2.12 & - \\
\hline Germacrene D & 1474 & 4.15 & 2.92 & 2.37 & 5.63 & 2.85 & 6.67 & 3.07 & 4.82 & 2.11 & 2.59 & 2.04 & 5.29 & 4.13 & 5.93 & 2.32 & 2.22 & 3.05 & - \\
\hline$\beta$-Selinene & 1476 & - & 0.46 & - & - & 0.71 & - & 0.64 & 0.65 & 0.71 & - & - & - & - & 1.15 & - & 0.48 & - & - \\
\hline Lyratyl isovalerate ${ }^{\mathrm{a}}$ & 1478 & - & - & 1.16 & - & - & - & - & - & 0.58 & - & - & - & - & - & - & - & - & - \\
\hline Bicyclogermacrene & 1482 & 2.87 & 2.21 & 1.71 & 4.05 & 2.2 & 6.16 & 1.46 & 2.69 & 2.08 & 3.04 & 1.18 & 2.56 & 2.44 & 5.63 & 2.39 & 1.53 & 1.95 & 1.48 \\
\hline Davana ether & 1491 & - & 4.43 & 4.56 & 0.65 & 1.09 & 1.21 & 0.82 & 2.46 & 0.62 & 6.23 & - & - & 3.02 & 2.55 & - & 1.59 & - & - \\
\hline Unknown 3 & 1494 & - & - & - & 0.8 & 0.44 & - & - & 0.38 & - & - & - & - & 0.54 & - & - & - & - & - \\
\hline Davana ether isomer & 1523 & - & 12.53 & 11.59 & 1.34 & 3.24 & 3.71 & 1.97 & 6.61 & 1.72 & 15.94 & - & - & 8.42 & 5.83 & - & 4.69 & - & - \\
\hline Davana ether isomer & 1554 & - & 6.62 & 6.13 & - & 1.65 & 1.44 & 0.81 & 3.41 & 0.85 & 7.27 & - & - & 4.5 & 2.87 & - & 2.4 & - & - \\
\hline Nerolidol & 1570 & - & - & 0.9 & - & - & - & - & - & - & 0.91 & - & - & - & 3.46 & - & - & - & - \\
\hline Spathulenol & 1576 & 1.09 & 1.12 & - & 0.96 & 1.28 & 3.74 & 3.74 & 0.99 & 1.39 & 0.55 & 1.36 & 1.02 & 1.42 & 3.1 & 0.99 & 0.85 & 1.9 & 2.06 \\
\hline Globulol $^{\mathrm{a}}$ & 1583 & - & - & - & - & - & 1.41 & 0.64 & 0.55 & - & - & - & - & 0.48 & 1.77 & - & - & - & - \\
\hline Caryophyllene oxide & 1587 & - & - & - & - & - & - & - & - & - & 1.03 & - & - & - & - & - & - & - & - \\
\hline$\beta$-Copaen-4- $\alpha$-ol ${ }^{\mathrm{a}}$ & 1590 & - & - & 0.8 & - & - & - & - & - & - & 0.45 & - & - & - & - & - & 1.05 & - & - \\
\hline Epiglobulol $^{\mathrm{a}}$ & 1593 & 0.69 & - & - & - & - & 2.08 & - & - & - & - & - & 0.57 & - & 0.82 & - & - & - & - \\
\hline Davanone & 1608 & - & 20.14 & 11.03 & 6.3 & 16.94 & 7.95 & - & 7.07 & 2.37 & 9.82 & - & - & 13.37 & 4.02 & - & 6.35 & - & - \\
\hline Rosifoliol $^{\mathrm{a}}$ & 1611 & - & - & - & - & - & - & - & - & - & - & - & - & - & 1 & - & - & - & - \\
\hline ester $^{\mathrm{a}}$ & 1619 & - & - & - & - & - & 0.94 & - & - & - & - & - & - & 0.74 & - & - & - & - & - \\
\hline$\alpha$-Acorenol & 1622 & - & - & 0.57 & - & 0.69 & 1.09 & 0.94 & - & - & 0.44 & - & 0.95 & - & 2.48 & - & 0.6 & - & - \\
\hline$\tau$-Cadinol & 1638 & - & - & - & - & 0.53 & 1.42 & 1.3 & - & - & - & - & 0.74 & - & 3.46 & - & - & - & - \\
\hline 3-Hydroxyisodavanone & 1644 & - & 4.96 & 5.23 & - & 1.06 & 1.12 & 2.5 & 4.8 & 4.74 & 5.41 & - & - & 4.39 & 2.97 & - & 4.29 & - & - \\
\hline$\beta$-Eudesmol & 1650 & - & - & - & - & - & 0.97 & 0.68 & - & - & - & - & - & - & - & - & - & - & - \\
\hline Unknown 4 & 1655 & - & - & - & - & - & - & - & 1.73 & - & - & - & - & - & 2.77 & - & - & - & - \\
\hline$\alpha-$ Cadinol $^{\mathrm{a}}$ & 1657 & 0.57 & 1.67 & 1.39 & - & 1.67 & 1.68 & 1.1 & 1.53 & - & 1.41 & - & - & 2.45 & 1.54 & - & 1.09 & - & - \\
\hline Selin-11-en-4- $\alpha$-ol & 1659 & - & - & - & - & - & - & - & 1.09 & - & - & - & - & - & 1.56 & - & - & - & - \\
\hline Xanthoxylin & 1661 & - & - & - & - & 0.74 & - & 1.22 & - & - & - & - & - & - & - & - & 0.58 & 2.13 & - \\
\hline (E)-Dihydrofarnesol ${ }^{\mathrm{a}}$ & 1672 & - & - & - & - & 1.12 & 0.85 & - & - & - & - & - & - & 0.64 & - & - & - & - & - \\
\hline Unknown 5 & 1679 & - & - & - & - & - & 0.58 & - & - & - & - & - & - & - & 0.59 & - & - & - & - \\
\hline Unknown 6 & 1681 & - & 3.37 & 3.11 & - & 1.75 & 1.46 & - & 1.61 & - & 4.22 & - & - & 2.18 & 0.87 & - & 1.13 & - & - \\
\hline isodavanone $^{\mathrm{a}}$ & 1692 & - & 3.56 & 2.37 & 1.38 & 2.89 & 2.12 & - & 1.52 & 1.69 & 2.11 & - & - & 3.46 & 0.79 & - & 1.88 & - & - \\
\hline$\beta$-Savanone-2-ol & 1722 & - & 0.79 & - & - & 0.87 & - & - & - & - & - & - & - & - & 0.84 & - & - & - & - \\
\hline Phytol $^{\text {a }}$ & 2112 & 0.52 & - & - & - & - & - & - & - & - & - & - & - & - & 0.52 & - & - & - & - \\
\hline Total & & 99.86 & 99.36 & 97.77 & 99.86 & 99.65 & 99.22 & 99.66 & 99.75 & 99.23 & 98.57 & 99.9 & 99.86 & 99.81 & 97.55 & 99.87 & 98.95 & 98.12 & 95.16 \\
\hline Total identified & & 99.86 & 95.56 & 93.78 & 98.69 & 96.41 & 96.76 & 98.83 & 95.7 & 99.23 & 93.37 & 99.9 & 99.86 & 96.63 & 93.32 & 99.87 & 97.11 & 98.12 & 95.16 \\
\hline Monoterpenes hydrocarbons & & 6.82 & 0.7 & 2.29 & 1.94 & 3.64 & 2.21 & 4.78 & 3.02 & 1.08 & 2 & 4.88 & 6.69 & 1.83 & 0.43 & 5.64 & 3.98 & 3.34 & 2.95 \\
\hline Oxygenated monoterpenes & & 82.93 & 32.59 & 41.14 & 76.09 & 53.71 & 48.42 & 72.47 & 53.83 & 79.29 & 31.5 & 89.91 & 81.82 & 44.72 & 39.07 & 87.79 & 64.11 & 87.52 & 88.67 \\
\hline Total monoterpenes & & 89.75 & 33.29 & 43.43 & 78.03 & 57.35 & 50.63 & 77.25 & 56.85 & 80.37 & 33.5 & 94.79 & 88.51 & 46.55 & 39.5 & 93.43 & 68.09 & 90.86 & 91.62 \\
\hline Sesquiterpenes hydrocarbons & & 7.02 & 6.45 & 4.08 & 9.68 & 5.76 & 14.4 & 6.88 & 8.59 & 4.9 & 7.79 & 3.22 & 7.85 & 7.19 & 14.24 & 4.71 & 4.23 & 5 & 1.48 \\
\hline Oxygenated sesquiterpenes & & 2.35 & 55.82 & 45.73 & 10.63 & 33.03 & 31.73 & 14.5 & 30.03 & 13.96 & 51.57 & 1.36 & 3.28 & 42.89 & 39.06 & 0.99 & 24.79 & 1.9 & 2.06 \\
\hline Total sesquiterpenes & & 9.37 & 62.27 & 49.81 & 20.31 & 38.79 & 46.13 & 21.38 & 38.62 & 18.86 & 59.36 & 4.58 & 11.13 & 50.08 & 53.3 & 5.7 & 29.02 & 6.9 & 3.54 \\
\hline
\end{tabular}


Figure 1. Origins of Artemisia herba-alba plants.
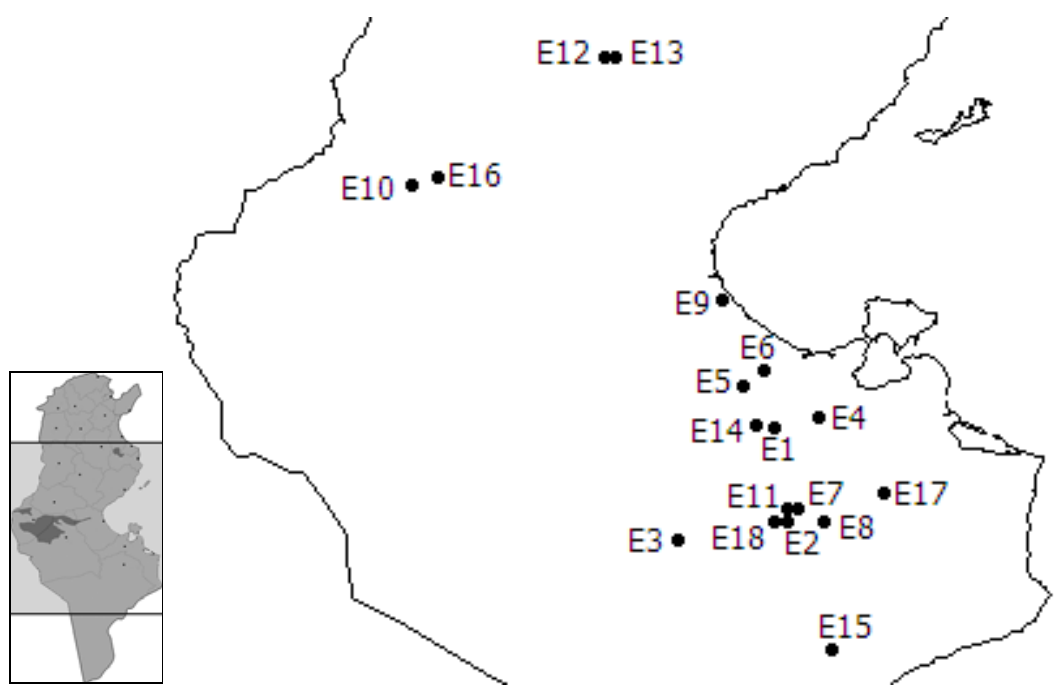

\section{Experimental}

\subsection{Plant material}

A. herba-alba aerial parts (leaves and stems) were collected from subcultured plants originated from different localities in sub-arid to Saharan domains of Tunisia (Figure 1) at the Institut des Régions Arides Médenine. Plant identification was carried out by A. Ferchichi, botanist at the Institut des Régions Arides Médenine. All samples were shade dried for 15 days at room temperature with ventilation. The material was cut into small pieces and subjected to hydrodistillation using a Clevenger-type apparatus for 4 hours. The oil was collected and stored at $-12{ }^{\circ} \mathrm{C}$ in amber vials before analysis.

\subsection{GC analysis}

Analytical gas chromatography was carried out using an Agilent-HP6890 gas chromatography system fitted with a DB-5 fused silica capillary column $(30 \mathrm{~m} \times 0.32 \mathrm{~mm}, 0.25 \mu \mathrm{m}$ film thickness). Carrier gas was helium at a flow rate of $1 \mathrm{~mL} / \mathrm{min}$. Column temperature was initially kept at $60^{\circ} \mathrm{C}$ for $5 \mathrm{~min}$, then gradually increased to $280^{\circ} \mathrm{C}$ at a rate of $2^{\circ} \mathrm{C} / \mathrm{min}$, and held for $10 \mathrm{~min}$. Samples $(1 \mu \mathrm{L}$, appropriately diluted in hexane) were injected at $280^{\circ} \mathrm{C}$ with split mode (1:50). A hydrocarbon mixture for retention index (RI) measurement was injected at the same above conditions. Flame ionization detector (FID) was set at $280^{\circ} \mathrm{C}$.

\subsection{GC-MS analysis}

GC-MS analysis was carried on an Agilent-HP6890 gas chromatography system (same specifications and conditions as above), coupled with a high resolution Waters Micromass Autospec 
Ultima mass detector operating in the EI mode $(70 \mathrm{eV})$. Components identification was done by GC (according to RI) and GC-MS (according to fragmentation patterns) and by comparing results with literature [28-30]. Retention indices (RI) were calculated by comparing the retention times of the eluting peaks with those of standard hydrocarbon mixture using a Van Den Dool and Kratz formula [31]. The components concentration was calculated from GC peak areas without the use of correction factors.

\section{Conclusions}

This study revealed a high level of chemical polymorphism of the essential oils of $A$. herba-alba originated from different localities in southern Tunisia. Ten samples had a chemical composition similar to that published for A. herba-alba oils from different countries but eight samples had an original chemical composition. This remarkable polymorphism could be due to differences in the ecological and climatic characteristics of the localities from where samples were taken.

\section{References and Notes}

1. Quezel, P.; Santa, S. New flora of Algeria and meridional desertic regions (in French). CNRS: Paris, France, 1962.

2. Zohary, M. Geobotanical foundations of the Middle East. Gustav Fisher Verlag Swets \& Zeitlinger: Stuttgart, Germany, 1973.

3. Breckle, S.W. Temperate deserts and semi-deserts of Afghanistan and Iran. Elsevier Scientific Publishing Company: New York, NY, USA, 1983. pp. 271-316.

4. LeFloc'h, E.; Schoenenberger, A.; Nabli, A.; Valdeyron, M.A. Biology and ecology of principal taxons (in French). Faculty of Sciences of Tunis; Official printing office of the Tunisian republic: Tunisia, 1989. pp. 49-193.

5. Nabli, M.A. Elements of botany and phyto-ecology (in French). Faculty of Sciences of Tunis; Official printing office of the Tunisian republic: Tunisia, 1989.

6. Bailey, C.; Danin, A. Bedouin plant utilization in Sinai and Negev. Econ. Bot. 1981, 35, 145-162.

7. Jouad, H.; Haloui, M.; Rhiouani, H.; Hilaly, J.E.; Eddouks, M. Ethnobotanical survey of medicinal plants used for the treatment of diabetes, cardiac and renal diseases in the North centre region of Morocco (Fez-Boulemane). J. Ethnopharmacol. 2001, 77, 175-182.

8. ElKar, C. Aromatic plants of south Tunisia: inventory, characterization and essential oil yield (in French). Faculty of Sciences of Tunis: Tunisia, 2003.

9. Oppenheimer, H.R. Drought adaptation: xerophytisme. UNESCO: Paris, France, 1961; pp. 115153.

10. Ourcival, J.M. Response of two chamephytes from presaharan Tunisia to different perturbations and constraints (in French).USTL: Montpellier, France, 1992.

11. Ferchichi, A. Contribution to cytotaxonomic and biologic study of Artemisia herba-alba Asso in presaharan Tunisia (in French). Acta Bot. Gallica 1997, 144, 145-154.

12. Yashphe, J.; Segal, R.; Breuer, A.; Erdreich-Naftali, G. Antibacterial activity of Artemisia herbaalba. J. Pharm. Sci. 1979, 68, 924-925. 
13. Al-Shamaony, L.; Al-Khazraji, S.M.; Twaij, H.A. Hypoglycemic effect of Artemisia herba alba. II. Effect of a valuable extract on some blood parameters in diabetic animals. J. Ethnopharmacol. 1994, 43, 167-171.

14. Al-Khazraji, S.M.; al-Shamaony, L.A.; Twaij, H.A. Hypoglycemic effect of Artemisia herbaalba. I. Effect of different parts and influence of the solvent on hypoglycaemic activity. $J$. Ethnopharmacol. 1993, 40, 163-166.

15. Hatimi, S.; Boudouma, M.; Bichichi, M.; Chaib, N.; Idrissi, N.G. Evaluation in vitro of antileishmanien activity of Artemisia herba-alba Asso (in French). Presented at Franco-African meeting of pediatrics $\mathrm{N}^{\circ} 14$, Paris, France, 2000; Exotic pathology society: Paris, France, 2000; pp. 57-70.

16. Feuerstein, I.; Danin, A.; Segal, R. Constitution of the essential oil from an Artemisia herba-alba population of Spain. Phytochemistry 1988, 27, 433-434.

17. Salido, S.; Valenzuela, L.R.; Altarejos, J.; Nogueras, M.; Sanchez, A.; Cano, E. Composition and infraspecific variability of Artemisia herba-alba from southern Spain. Biochem. Syst. Ecol. 2004, 32, 265-277.

18. Feuerstein, I.; Müller, D.; Hobert, K.; Danin, A.; Segal, R. The constitution of essential oils from Artemisia herba-alba populations of Israel and Sinaï. Phytochemistry 1986, 25, 2343-2347.

19. Hudaib, M.; Aburjai, T. Composition of the Essential Oil from Artemisia herba-alba Grown in Jordan. J. Essen. Oil Res. 2006, 18, 301-304.

20. Lamiri, A.; Belanger, A.; Berrada, M.; Zrira, S.; Benjilali, B. Chemical polymorphism of Artemisia herba-alba Asso from Morocco (in French); Rabat: Morocco, 1997.pp. 69-79.

21. Lamiri, A.; Belanger, A.; Berrada, M.; Ismaïli-Alaoui, M.M.; Benjilali, B. Origin of chemical polymorphism of Moroccan Artemisia herba-alba Asso(in French); Rabat: Morocco, 1997.pp. 8192.

22. Vernin, G.; Merad, O.; Vernin, G.M.F.; Zamkotsian, R.M.; Parkanyi, C., GC-MS analysis of Artemisia herba-alba Asso essential oils from Algeria. In Food Flavors: Generation, Analysis, and Process Influence; Elsevier Science: BV, Greece, 1995; pp. 147-205.

23. Dob, T.; Benabdelkader, T. Chemical Composition of the Essential Oil of Artemisia herba-alba Asso Grown in Algeria. J. Essen. Oil Res. 2006, 18, 685-690.

24. Akrout, A. Essential oil study of some pastoral plants from Matmata (south Tunisia) (in French). Cah. Options Med. 2004, 62, 289-292.

25. Neffati, A.; Skandrani, I.; Sghaier, M.B.; Bouhlel, I.; Kilani, S.; Ghedira, K.; Neffati, M.; Cherif, I.; Hammami, M.; Chekir-Ghedira, L. Chemical composition, mutagenic and antimutagenic activities of essential oils from (Tunisian) Artemisia campestris and Artemisia herba-alba. J. Essen. Oil Res. 2008, 20, 471-477.

26. Soliman, M.M.M. Phytochemical and toxicological studies of Artemisia L. (compositae) essential oil against some insect pests. Arch. Phytopathol. Plant Prot. 2007, 40, 128-138.

27. Sbouï, S. Comparative study of active substances and minerals from some Artemisia herba-alba Asso populations from south Tunisia (in French). Faculty of Sciences of Tunis: Tunisia, 2002.

28. Appendino, G.; Gariboldi, P.; Nano, G.M.; Tetenyi, P. Tetrahydrofuran-type terpenoids from Tanacetum vulgare. Phytochemistry 1984, 23, 2545-2551. 
29. Adams, R.P. Identification of essential oil components by gas chromatography/quadrupole mass spectrometry, $2^{\text {nd }}$ Ed.; Allured Publ. Corp.: Carol Stream, IL: USA, 2001.

30. Weyerstahl, P.; Marschall, H.; Schröder, M.; Wahlburg, H.C.; Kaul, V.K. The sesquiterpene fraction of the essential oil of Artemisia laciniata Willd. Flavor Frag. J. 1997, 12, 315-325.

31. Van Den Dool, H.; Kratz, R.D. A generalization of the retension index system including linear temperature programmed gas-liquid partition chromatography. J. Chromatogr. 1963, 11, 463-471.

Sample Availability: Samples of the compounds are available from the authors.

(C) 2009 by the authors; licensee Molecular Diversity Preservation International, Basel, Switzerland. This article is an open-access article distributed under the terms and conditions of the Creative Commons Attribution license (http://creativecommons.org/licenses/by/3.0/). 